\title{
Pharmacological Activation of RXR-a Promotes Hematoma Absorption in a PPAR-y-Dependent Pathway After Intracerebral Hemorrhage
}

\section{Chaoran Xu}

Zhejiang University School of Medicine Second Affiliated Hospital

\section{Huaijun Chen}

Zhejiang University School of Medicine Second Affiliated Hospital

\section{Shengjun Zhou}

Zhejiang University School of Medicine Second Affiliated Hospital

Chenjun Sun

Zhejiang University School of Medicine Second Affiliated Hospital

\section{Xiaolong Xia}

Zhejiang University School of Medicine Second Affiliated Hospital

\section{Yucong Peng}

Zhejiang University School of Medicine Second Affiliated Hospital

\section{Jianfeng Zhuang}

Zhejiang University School of Medicine Second Affiliated Hospital

\section{Xiongjie Fu}

Zhejiang University School of Medicine Second Affiliated Hospital Hanhai Zeng

Zhejiang University School of Medicine Second Affiliated Hospital Hang Zhou

Zhejiang University School of Medicine Second Affiliated Hospital

\section{Yang Cao}

Zhejiang University School of Medicine Second Affiliated Hospital

\section{Qian Yu}

Zhejiang University School of Medicine Second Affiliated Hospital

\section{Yin Li}

Zhejiang University School of Medicine Second Affiliated Hospital

\section{Libin Hu}

Zhejiang University School of Medicine Second Affiliated Hospital

\section{Guoyang Zhou}

Zhejiang University School of Medicine Second Affiliated Hospital

\section{Feng Yan}


Zhejiang University School of Medicine Second Affiliated Hospital

\section{Gao Chen ( $\sim$ d-chengao@zju.edu.cn )}

Zhejiang University School of Medicine Second Affiliated Hospital https://orcid.org/0000-0003-10850028

\section{Jianru Li ( $\sim$ lijianru@zju.edu.cn )}

Zhejiang University School of Medicine Second Affiliated Hospital

\section{Research}

Keywords: Intracerebral hemorrhage, RXR-a, PPAR-y, polarization, phagocytosis, neuroinflammation Posted Date: February 18th, 2021

DOl: https://doi.org/10.21203/rs.3.rs-67854/v2

License: (c) (i) This work is licensed under a Creative Commons Attribution 4.0 International License. Read Full License 


\section{Abstract}

Endogenously eliminating the hematoma is a favorable strategy in addressing intracerebral hemorrhage (ICH). This study sought to determine the role of retinoid X receptor-a (RXR-a) in the context of hematoma absorption after $\mathrm{ICH}$. Our results show pharmacologically activating RXR-a with bexarotene significantly accelerated hematoma clearance and alleviated neurological dysfunction after ICH. RXR-a was expressed in the microglia/macrophages, neurons, and astrocytes. Mechanistically, bexarotene promoted the nuclear translocation of RXR- $\alpha$ and PPAR- $\gamma$, alongside reducing neuroinflammation by modulating microglia/macrophages reprograming into the M2 phenotype from the M1 phenotype. However, all the beneficial effects of RXR-a in ICH were reversed by the PPAR-y inhibitor GW9662. In conclusion, the pharmacological activation of RXR-a conferred robust neuroprotection against ICH by accelerating hematoma clearance and repolarizing microglia/macrophages towards the M2 phenotype through PPAR$\mathrm{y}$-related mechanisms. Our data support the notion that RXR-a might be a promising therapeutic target for $\mathrm{ICH}$.

\section{Introduction}

Intracerebral hemorrhage $(\mathrm{ICH})$ is one of the most fatal cerebrovascular diseases and is linked to high morbidity and mortality rates [1, 2]. Primary brain injury caused by ICH usually occurs within the first few hours of onset and hematoma mechanically damages the adjacent tissues. Subsequently, hematomainduced secondary brain injuries, including inflammatory responses, microglia activation, oxidative stress, and neuronal apoptosis and necrosis occurring after the primary injury can result in severe neurological deficits and death [1,3]. A growing body of studies indicates that hematoma clearance may represent a unique target in ICH treatment [4]. Although the early removal of hematoma using surgical procedures is underway, yielding promising results, this shows unsatisfactory effects in neurological recovery and fail to address the secondary brain damage of $\mathrm{ICH}$ [5-7]. Therefore, new therapeutic strategies focusing on endogenous hematoma clearance for $\mathrm{ICH}$ are urgently needed.

In the central nervous system (CNS), microglia/macrophages serve as the main phagocytes involved in the defense against brain damage, including $\mathrm{ICH}$, through the phagocytosis of red blood cells and the removal of tissue debris $[4,8,9]$. Microglia/macrophages can be activated by hematoma components, such as thrombin and neurotoxins after tissue damage occurs [10]. It has been found that microglia/macrophages of the brain may dynamically be activated into two polarized states, termed the classical M1-like phenotype and the alternative M2-like phenotype [11]. Alternatively activated M2-like microglia/macrophages of the brain are considered to be anti-inflammatory and are generally involved in phagocytosis, and tissue repair [3]. The evidence above suggests that promoting the resolution of inflammation and increasing phagocytic activity by inhibiting the M1 phenotype and promoting the M2 phenotype may be an effective and promising therapeutic strategy for $\mathrm{ICH}$ as well.

Retinoid X receptors (RXRs) are members of the nuclear receptors superfamily $[12,13]$. Currently, there are three different RXR isoforms ( $a, \beta$, and $\gamma$ ). RXR- $a$ is highly expressed in all human and rodent 
macrophage-type cells (brain microglia, liver Kupffer cells, and bone osteoclasts). Once activated, RXR-a translocates into the nucleus and regulate the transcription of target genes, and is involved with multiple cellular processes, such as monocyte/macrophage differentiation, phagocytosis, and metabolism [13-16]. Current studies reported that activation of RXR-a, which forms a heterodimer with peroxisome proliferatoractivated receptor- $\gamma$ (PPAR- $\gamma$ ), was implicated as a promising treatment for Alzheimer's disease [16, 17]. Notably, a recent study has demonstrated that the activation of RXR-a may promote the nuclear accumulation and transcriptional activity of PPAR- $y$, therefore modulating microglia polarization in traumatic brain injury [18]. However, the role of RXR-a in the pathological process following ICH has not yet been fully understood.

Based on the evidence above, we aimed to determine the role of RXR-a in modulating the polarization of microglia/macrophages and promoting hematoma clearance and neurological function via the PPAR- $Y$ pathway.

\section{Materials And Methods}

\section{Animals and ethical statement}

All procedures were approved by the ethics committee of Zhejiang University and followed the National Institutes of Health guidelines for the Care and Use of Laboratory Animals. Adult male C57BL/6J mice (age 8-10 weeks, weight 22-25 g, n=409) were obtained from SLAC Laboratory Animal Co., Ltd. (Shanghai, China). The mice were kept in a humidity-controlled room $\left(25 \pm 1{ }^{\circ} \mathrm{C}, 12\right.$-h light/dark cycle) and were raised with free access to food and water.

\section{ICH model}

The autologous blood injection model of ICH was performed as previously described [19]. Briefly, mice were anesthetized and maintained under $1 \%$ pentobarbital sodium, after which a volume of $25 \mu \mathrm{L}$ of autologous blood was injected $2.5 \mathrm{~mm}$ to the right and $3 \mathrm{~mm}$ below the bregma at a $5^{\circ}$ angle toward the midline. Mice in the sham group underwent the same procedure (anesthesia and needle insertion), except for injection.

\section{Experimental design}

All mice were randomly assigned to the following experiments (Figure 1). A total of 409 mice including the dead ones, were used in this study.

\section{Experiment I}

Bexarotene is a highly selective RXR-a agonist approved by The Food and Drug Administration as an antineoplastic agent for the treatment of cutaneous T-cell lymphoma, with high blood-brain barrier permeability and a good safety profile [20]. To assess the effects of RXR-a activation on hematoma clearance and neurological function after ICH, the RXR-a agonist bexarotene was used. Neurological 
function (as assessed by the cylinder test, corner turn test, and forelimb placement test) was tested after $\mathrm{ICH}$. Mice were assigned into three groups: sham group, ICH+vehicle group (10\% Dimethyl sulfoxide (DMSO), in saline), and $\mathrm{ICH}+$ bexarotene group $(5 \mathrm{mg} / \mathrm{kg})(\mathrm{n}=9)$. The sham group received the same volume of vehicle intraperitoneally at the same time points after $\mathrm{ICH}$ induction. A T2* weighted magnetic resonance imaging (MRI) scan was used to measure the hematoma volume at 1, 3, 7, 14, and 28 days after ICH. Mice were randomly divided into two groups: ICH+vehicle (10\% DMSO, in saline), and $\mathrm{ICH}+$ bexarotene $(5 \mathrm{mg} / \mathrm{kg})(\mathrm{n}=6)$.

\section{Experiment II}

To assess the expression pattern of RXR-a and PPAR-y after ICH, mice were assigned to five groups: a sham, ICH 1 d, ICH 3 d, ICH 7 d, and ICH 14 d group $(n=6)$. Whole-cell lysates Western blots $(n=6)$ and cytoplasmic and nuclear protein Western blots $(n=6)$ were performed at different time-points. Next, the cellular location of RXR-a was assessed by double immunofluorescence staining in ICH (3 days) group $(n=6)$. To assess the nuclear translocation of RXR-a, mice were divided into three groups: a sham group, $\mathrm{ICH}+$ vehicle group (10\% DMSO, in saline), and $\mathrm{ICH}+$ bexarotene group $(\mathrm{n}=6)$. Whole-cell lysate Western blots $(n=6)$ and cytoplasmic and nuclear protein Western blots $(n=6)$ in these groups were performed at 3 days after ICH. To determine whether PPAR-y plays a role in RXR- $a$ activation after ICH, the RXR- $a$ agonist bexarotene and PPAR-y antagonist GW9662 were used. To assess the nuclear translocation of PPAR- $\gamma$, mice were divided into four groups: a sham group, $\mathrm{ICH}+$ vehicle group (10\% DMSO, in saline), $\mathrm{ICH}+$ bexarotene+vehicle group, and an $\mathrm{ICH}+$ bexarotene+GW9662 $(4 \mathrm{mg} / \mathrm{kg})(\mathrm{n}=6)$. Whole-cell lysate Western blots $(n=6)$ and cytoplasmic and nuclear protein Western blots $(n=6)$ in these groups were performed at 3 days after $\mathrm{ICH}$.

\section{Experiment III}

To assess the mechanism underlying the role of RXR-a activation in $\mathrm{ICH}$, mice were randomly assigned to four groups: a sham group, ICH+vehicle group (10\% DMSO, in saline), $\mathrm{ICH}+$ bexarotene group+vehicle group (10\% DMSO, in saline), and an ICH+bexarotene (5 mg/kg)+GW9662 (4 mg/kg) group. Immunofluorescence staining $(n=4)$, Western blots $(n=6)$, and enzyme-linked immunosorbent assays (ELISAs) $(n=6)$ were used to assess the polarization of microglia/macrophages and PPAR-y transcription activity. To study whether microglia had played a major role, mice were divided into four groups: $\mathrm{ICH}+$ Clophosome $(5 \mu \mathrm{L})+$ vehicle $(10 \% \mathrm{DMSO}$, in saline) group, $\mathrm{ICH}+\mathrm{Clophosome}(5 \mu \mathrm{L})+$ bexarotene $(5$ $\mathrm{mg} / \mathrm{kg}$ ) group, $\mathrm{ICH}+$ control liposome $(5 \mu \mathrm{L})+$ vehicle(10\% DMSO, in saline) group, $\mathrm{ICH}+$ control liposome (5 $\mu \mathrm{L})$ +bexarotene $(5 \mathrm{mg} / \mathrm{kg})$ group $(\mathrm{n}=6)$. A total of 6 mice were used to determine the efficiency of microglia/macrophages depletion: $\mathrm{ICH}+$ Clophosome $(5 \mu \mathrm{L})$ group, $\mathrm{ICH}+\mathrm{Clophosome}(5 \mu \mathrm{L})$ group $(\mathrm{n}=6)$.

\section{Experiment IV}

Next, we assessed whether PPAR-y plays a role in the protective effects linked to RXR-a activation. To this end, the cylinder test, corner turn test, and forelimb placement test were used after $\mathrm{ICH}$. Mice were randomly divided into three groups: an $\mathrm{ICH}+$ vehicle group (10\% DMSO, in saline), $\mathrm{ICH}+$ bexarotene+vehicle 
group ( $5 \mathrm{mg} / \mathrm{kg}), \mathrm{ICH}+$ bexarotene $(5 \mathrm{mg} / \mathrm{kg})+\mathrm{GW} 9662(4 \mathrm{mg} / \mathrm{kg})(\mathrm{n}=6)$. The sham group received the same volume of vehicle intraperitoneally at the same time points after ICH induction. An MRI scan was used to measure the hematoma volume at $1,3,7$, and 14 days after $\mathrm{ICH}$. Mice were randomly divided into three groups: an ICH+vehicle group (10\% DMSO, in saline), ICH+bexarotene+vehicle group $(5 \mathrm{mg} / \mathrm{kg})$, and $\mathrm{ICH}+$ bexarotene $(5 \mathrm{mg} / \mathrm{kg})+\mathrm{GW} 9662(4 \mathrm{mg} / \mathrm{kg})$ group $(\mathrm{n}=6)$.

\section{Drug administration}

Bexarotene (MedChem Express, New Jersey, USA) was dissolved in 10\% dimethyl sulfoxide (DMSO) as previously described [21]. The selective PPAR-y antagonist GW9662 (MedChem Express, New Jersey, USA) was diluted in $10 \%$ DMSO. Bexarotene solution ( $5 \mathrm{mg} / \mathrm{kg}$ ) or an equal volume of vehicle or bexarotene solution $(5 \mathrm{mg} / \mathrm{kg})+$ GW9662 solution $(4 \mathrm{mg} / \mathrm{kg}$ ) was administered intraperitoneally $1 \mathrm{~h}$ after $\mathrm{ICH}$ for the first time, followed by daily injections until sacrifice. For depletion of microglia and macrophage, mice were injected with $25 \mu \mathrm{L}$ of blood mixed with $5 \mu \mathrm{L}$ anionic forms Clophosome or control liposomes (FormuMax, CA, USA). The dosage and time points of bexarotene, GW9662, and Clophosome were based on a previous study [22-24].

\section{Calculation of hematoma volume}

Mice were anesthetized with $1 \%$ pentobarbital sodium for the MRI examination. An MRI was performed on days $1,3,7,14$, and 28 after ICH in a 3.0-T MRI scanner. The MRI included a T2* sequence. The scanning parameters for T2* weighted imaging were: TR/TE $=2200 / 103.8 \mathrm{~ms}$, Number of averages $=10$, acquisition matrix $=208 \times 208$, voxel size $=0.12 \times 0.12 \times 1 \mathrm{~mm}$, flip angle $=130^{\circ}$, slices $=5$. MRI image datasets were obtained in the Digital Imaging and Communications in Medicine (DICOM) format. The data were transformed into the NIfTI (.nii) format and then assessed with 3D Slicer. The 3D-Slicer method is one of the software methods serving to measure the volume of a hematoma(http://www.slicer.org/). Hematomas were manually identified pixel by pixel in each slice. Next, a 3D model was established and the hematoma volume was calculated by adding up the volume of the pixels. Alternatively, hematoma hemoglobin contents were used to quantify the hematoma volume [25]. Four $1 \mathrm{~mm}$ coronal brain slices in the bleeding area were collected and homogenized and extracted ultrasonically with $300 \mu \mathrm{l}$ distilled water. Different volumes of mouse autologous blood $(0,1.0,2.0,4.0$, and $8.0 \mu \mathrm{l})$ were added to $300 \mu \mathrm{l}$ of normal brain tissue lysate to generate a standard curve. After centrifugation at $12,000 \mathrm{~g}$ for 30 minutes, the supernatant was collected, and $80 \mu$ l of Drabkin's reagent (Sigma-Aldrich, MO, USA) was added to 20 $\mu \mathrm{l}$ of supernatant and incubated in a 96-well plate at room temperature for 15 minutes. The absorbance of the solution was measured at a wavelength of $540 \mathrm{~nm}$.

\section{Behavioral tests}

Neurobehavioral functions were evaluated by a forelimb placing test, forelimb use asymmetry (cylinder) test, and a corner turn test, as previously reported [20]. Baseline data were recorded for the reduction of variability and identification of the preferential side. The neurological scores were evaluated by a blinded observer. 
Forelimb placement can be assessed by stimulating the mouse's vibrissae to trigger a response. To test the function of the forelimbs, the researchers held the animal's torso and hung the forelimbs freely, while brushing its vibrissae on the corner edge of a table. Non-brain-damaged animals usually respond to placing the forelimb on the table on the same side as the affected side, while ICH mice will be impaired in the placement of their paws. This test can be scored by counting the percentage of placements.

For the forelimb use asymmetry test (cylinder test), the mouse is placed in a transparent cylinder and observed the independent wall contacts. The behavior score was recorded as the number of times the ipsilateral (unimpaired) forelimb (I), contralateral (impaired) forelimb (C), and both forelimbs (B). A single overall limb use asymmetry score was calculated as follows: Limb use asymmetry score $=[\mathrm{I} /(\mathrm{I}+\mathrm{C}+\mathrm{B})]$ $[\mathrm{C} /(\mathrm{I}+\mathrm{C}+\mathrm{B})]$.

For the corner test, a mouse was then placed between the boards facing the $30^{\circ}$ angle corner. As the mouse approaches the corner, both sides of the vibrissae were simultaneously stimulated causing the animal to rear and turn $180^{\circ}$, the number of the right turns was then calculated.

\section{Immunofluorescence double labeling}

Coronal sections were blocked with 5\% Bovine serum albumin (BSA) and 0.3\% Triton X-100 and then incubated with primary antibodies overnight at $4^{\circ} \mathrm{C}$. The primary antibodies used were mouse anti-NeuN (1:500, ab-104224, Abcam, Cambridge, UK), goat anti-Iba-1 (1:500, ab-5076, Abcam, Cambridge, UK), mouse anti-GFAP protein (1:500, ab10062, Abcam, Cambridge, UK), rabbit anti-RXR-alpha antibody (1:250, ab125001, Abcam, Cambridge, UK), rabbit anti-Arg1 (1:500, 16001-1-AP, Proteintech, Hubei, China), rabbit anti-Nitric oxide synthase (iNOS) Antibody (18985-1-AP,1:500, Proteintech, Hubei, China), and rabbit anti-PPAR-y antibody (1:250, ab178860, Abcam, Cambridge, UK). The sections were incubated with secondary antibodies at room temperature for $2 \mathrm{~h}$. Finally, the sections were observed and analyzed using a fluorescence microscope (Olympus, Tokyo, Japan). Photomicrographs were saved and merged using the Image-Pro Plus software. To assess nuclear fluorescence ratio, Image $\mathrm{J}$ software was used to determine the nuclear fluorescence and nuclear+cytoplasmic fluorescence as previously reported [26]. Briefly, the integrated density measurement of pixel numbers was made on an ROI consisting of the total nucleus and the whole cell (nucleus and cytoplasmic). For each group, six mice were evaluated, and each sample had three brain sections, with each section being examined under three random fields of vision to acquire the cells (48-60 cells per group were counted).

\section{Enzyme-linked immunosorbent assay}

Brain samples were homogenized in chilled lysis buffer containing protease and phosphatase inhibitor cocktails (P1005, Beyotime, Shanghai, China). ELISA kit for mouse TNF-a (Boster, EK0527, Wuhan, China) was used to assess the levels of TNF-a in the brain. The total protein content of each sample was determined by a bicinchoninic acid (BCA) assay (ThermoFisher, Waltham, MA USA). An equal amount of protein brain homogenates were diluted at a 1:10 ratio with the sample diluent provided with the kits, and all procedures were performed per the manufacturer's instructions. The PPAR-y activity was measured 
using PPAR- $y$ Transcription Factor Assay (ab133101, Abcam, Cambridge, UK). The protein concentrations were equal. The ELISA was performed following the manufacturer's instructions.

\section{Western blot analysis}

A Western blot analysis was performed as previously described [27]. Briefly, the basal ganglia were homogenized and centrifuged for $15 \mathrm{~min}\left(13,000 \mathrm{~g}, 4^{\circ} \mathrm{C}\right)$. For the whole cell lysates, tissue proteins from basal ganglia were lysed using RIPA lysis buffer. For the extraction of cytoplasmic and nuclear proteins, the cytoplasmic and nuclear proteins were extracted using Cytoplasmic and Nuclear Protein Extraction Kit (P0027, Beyotime, Shanghai, China). Proteins were assessed using a BCA Protein Assay Kit (Thermo Fisher Scientific, Waltham, MA USA). An equal amount of protein $(40 \mu \mathrm{g})$ was suspended in a loading buffer (denatured at $95^{\circ} \mathrm{C}$ for $5 \mathrm{~min}$ ), loaded on an SDS-PAGE, and transferred to nitrocellulose membranes. Next, the membranes were blocked with a nonfat dry milk buffer for $1 \mathrm{~h}$ and incubated overnight with the primary antibody. The membranes were then incubated with the secondary antibody for $1 \mathrm{~h}$ at room temperature. Bands were visualized using the ECL Plus chemiluminescence reagent kit (Amersham Bioscience, Arlington Heights, IL). The band densities were quantified using Image $\mathrm{J}$ software. The primary antibodies used in this study were rabbit anti-PPAR- $y$ (1:1000, Abcam, Cambridge, UK, ab45036), rabbit anti-RXR-a antibody (1:1000, ab125001, Abcam, Cambridge, UK), rabbit anti-Arg1 (1:1000, 16001-1-AP, Proteintech, Hubei, China), mouse anti-GAPDH (1:5000, ab8245, Abcam, Cambridge, UK), mouse anti- $\beta$ actin (1:5000, ab8227, Abcam, Cambridge, UK), and rabbit anti-histone H3 (1:2000, \#9715, Cell Signaling Technology, MA, USA).

\section{Statistical analysis}

All data in this study are expressed as the mean \pm standard error of the mean. For data that meet a normal distribution and homogeneity of variance, differences among the groups were analyzed using a one-way analysis of variance (ANOVA) followed by a Tukey's multiple comparison test. A Kruskal-Wallis test with a Bonferroni correction was used for the non-normally distributed data. All statistical analyses were performed in SPSS (version 22.0). A p-value $<0.05$ was considered statistically significant.

\section{Results}

\section{Animal mortality rate}

A total of 409 mice were used, of which 80 underwent a sham procedure and 329 mice underwent an ICH induction. None of the sham mice died, and the mortality rate in the ICH group was 9.7\% (32/329). There was no significant difference in mortality rate across the modeling groups.

\section{Pharmacological activation of RXR-a significantly alleviated neurological dysfunction and promoted hematoma clearance after ICH}

Bexarotene, an FDA-approved antineoplastic agent, which functions by selectively activating RXR-a, was introduced in the current study to determine the effects of RXR-a under ICH conditions. Three different 
behavioral tests, including cylinder test, corner turn test, and forelimb placement test, were used to assess the effects of RXR-a on behavioral recovery at days 1, 3, 7, 14, and 28 after ICH. There was no significant difference in the behavioral tests of mice in the four groups at baseline (pre-lCH). Animals in the modeling groups suffered from a serious neurological deficit at $24 \mathrm{~h}$ after $\mathrm{ICH}$ when compared with sham-operated mice (Fig. 2A-C). The activation of RXR-a significantly improved the neurological function in all the tests carried out on days 3 and 7 after $\mathrm{ICH}$, as compared to the vehicle group. A T2*-weighted MRI scan (coronal sections) was used to measure intracranial hematoma volume in parenchyma and ventricles at $1,3,7,14$, and 28 days after ICH. No significant differences in hematoma volume were noted at $24 \mathrm{~h}$ postmodeling, suggesting that the models yield consistent hematoma volumes. Compared with the vehicle group, the activation of RXR-a with bexarotene significantly decreased the hematoma volume from day 3 to day 7 after ICH (Fig. 2D).

\section{Bexarotene promoted nuclear translocation of RXR-a in microglia/macrophages}

Dual-label immunofluorescence staining showed that RXR-a was expressed in microglia/macrophages (Iba-1), neurons (NeuN), and astrocytes (GFAP) in the perihematomal region on the sham group and the third-day post-ICH (Fig. 3A). Then we noticed that the microglia/macrophages at day 3 after ICH had a more obvious nuclear signal and higher nucleoplasm ratio compared to the sham group (Fig. 3B). However, no significant changes in the ratio of cytoplasm and nuclei were observed in neurons and astrocytes (Fig. 3C, D). To observe the temporal profiles of RXR-a after ICH in mice, we examined their total and subcellular expression patterns by Western blotting on the 1st, 3rd, 7th, and 14th days after ICH. The expression patterns of RXR- $\alpha$ in whole-cell of the ipsilateral hemispheres did not show any significant differences across these groups (Fig. 3E). The cytoplasmic expression of RXR-a was significantly reduced on the 3rd and 7th day after ICH (Fig. 3F), whereas the nuclear level showed an increase on the 3rd and 7th day in ICH-injured mice as compared to the sham group (Fig. 3G). To further explore the effect of bexarotene on the nuclear-cytoplasmic shuttling of RXR-a, the expression of RXR-a was assessed in the ipsilateral basal ganglia at 3 days after ICH by Western blotting. The total expression of RXR-a did not show any significant changes across the three groups (Fig. 3H). The RXR-a expression level of the bexarotene group was increased in the nuclei and decreased in the cytoplasm as compared with the vehicle group (Fig. 3I, J). In sum, the nuclear translocation of RXR-a was induced after ICH, especially on microglia/macrophages, and the administration of bexarotene further enhanced the nuclear translocation of RXR-a.

\section{Depletion of microglia/macrophages inhibited bexarotene-mediated hematoma clearance}

To study whether microglia had played a major role in the bexarotene-mediated hematoma resolution, Clodronate liposome treatment was used to deplete microglia/macrophages. To verify the validity of depletion, the number of microglia/macrophages and astrocytes of the peri-hematoma area at day 3 after $\mathrm{ICH}$ were counted. The number of Iba1 positive cells was less in the Clophosome treatment group, reducing by $85.5 \%$ of the control group (Fig. 4A, B). But this treatment does not deplete astrocyte (Fig. 4C). Besides, we examined the effect of bexarotene treatment on the hematoma resolution after depletion 
of microglia/macrophages. Quantification of hemoglobin showed that Clophosome treatment slowed the absorption of hematoma, and there was no statistical difference between bexarotene treatment and vehicle. Consistent with our previous results, the hemoglobin quantification of the group treated with control liposome showed bexarotene accelerated hematoma clearance at day 3 after ICH (Fig. 4D, E).

\section{Activation of RXR-a with bexarotene enhanced the nuclear translocation and transcription activities of PPAR-Y}

To determine the nuclear-cytoplasmic translocation of PPAR- $y$ after $\mathrm{ICH}$, immunofluorescence staining was performed in the perihematomal region on the sham group and the third-day post-ICH (Fig. 5A). We noticed that nuclear translocation of PPAR- $y$ was prominent in the microglia/macrophages after ICH compared to the sham group (Fig. 5B-D). Besides, we tested the total and subcellular expressions of PPAR- $\gamma$ by Western blotting at days 1, 3, 7, and 14 after ICH. The endogenous expression of PPAR- $\gamma$ in the whole cells increased on the 3rd day and remained significantly higher on the 14th day(Fig. 5E). The expression of the PPAR-y in the cytoplasm was reduced on the 7th day, and the expression of the PPAR-y in nuclei increased on the 3rd and 7th days after ICH (Fig. 5F, G). To explore whether PPAR-y played a role in the process of RXR-a activation, the nuclear-cytoplasmic translocation of PPAR- $\gamma$ was assessed 3 days after ICH among the four experimental groups. The total expression of PPAR-y did not show any significant changes between the vehicle group, the bexarotene group, and the bexarotene + GW9662 group (Fig. 5H). The PPAR-y expression in the bexarotene group showed a remarkable reduction in the cytoplasm and was increased in nuclei as compared with the vehicle group, while the PPAR-y antagonist GW9662, in contrast, decreased the PPAR-y expression in nuclei and increase the PPAR-y expression in the cytoplasm (Fig. 5I, J). To examine the effect of bexarotene on PPAR-y transcription activity, we performed a PPAR- $y$ transcriptional activity assay. The transcriptional activity of PPAR- $\gamma$ was increased after $\mathrm{ICH}$, and bexarotene further increased transcription activity. However, the effect was abolished by GW9662 treatment (Fig. 5K).

\section{RXR- $a$ activation regulated microglia/macrophages polarization through PPAR-y}

To determine whether the PPAR- $y$ played a role in the polarization of microglia/macrophages, we examined microglia/macrophage markers of polarization iNOS (a pro-inflammatory state (M1) marker) and Arg1 (an anti-inflammatory state (M2) marker) on day 3 after ICH by immunofluorescence. It was found that the expression of iNOS and Arg1 were all increased after ICH compared to the sham group (Fig. 6A, B). Furthermore, the expression of iNOS and Arg1 was high in Iba1+ cells (microglia/macrophages) in the perihematomal area of mouse brain slices. The co-expression of iNOS and Iba1 was less pronounced in the presence of bexarotene while GW9662 reversed this effect (Fig. 6A). In contrast, bexarotene led to a significant increase in the number of M2-like polarized microglia/macrophages, which was reversed by GW9662 administration (Fig. 6B). The ELISA revealed that the TNF-a levels were increased after ICH (Fig. 6C). Bexarotene reduced TNF-a, and the effect was attenuated by GW9662 (Fig. 6C). The expression of the M2-like marker Arg1 was measured by Western blot. Bexarotene stimulated the M2-like marker while GW9662 reversed this reduction (Fig. 6D). 


\section{Role of PPAR-y on RXR-a-mediated neuroprotection and hematoma absorption against ICH}

In the next part of this study, the PPAR-y antagonist GW9662 was introduced to further determine the role of PPAR-y in RXR-a-mediated protective effects under ICH conditions. Three behavioral tests were used to examine the role of PPAR-y on behavioral recovery on days $1,3,7,14$, and 28 after ICH. The activation of RXR-a significantly improved the neurological function, while the protective effects of the bexarotene treatment were reversed by PPAR-y inhibition (Fig. 7A-C). A T2*-weighted MRI scan (coronal sections) was used to measure the hematoma volume on days $1,3,7$, and 14 after ICH. Similarly, RXR-a activation significantly promoted hematoma absorption on days 3 and 7 after ICH (Fig. 7D, E). However, the blockade of PPAR-y with GW9662 reversed bexarotene-mediated hematoma absorption (Fig. 7D, E).

\section{Discussion}

In this present study, we investigated the role of RXR-a in the pathological process following ICH and assessed the relevant underlying mechanisms. Our findings are as follows: (1) The activation of RXR-a alleviated ICH-induced neurological deficits and promote hematoma absorption. (2) RXR-a nuclear translocation was observed after $\mathrm{ICH}$, and this effect was further enhanced by the pharmacological activation of RXR-a. (3) The hematoma clearance effect mediated by RXR- $a$ activation was attenuated after microglia/macrophages depletion. (4) A similar nuclear translocation of PPAR- $y$ following injury was also observed. Moreover, RXR- $\alpha$ activation could enhance PPAR- $y$ translocation, whereas the inhibition of PPAR-y reversed this effect. (5) RXR-a activation could tend to make microglia/macrophages polarize into M2-like rather than M1-like cells through PPAR- $y$ signaling. (6) The PPAR-y antagonist GW9662 abolished the protective effect of RXR- $\alpha$ activation after ICH. Based on the evidence above, the pharmacological activation of RXR-a significantly alleviated ICH-induced neurological deficits and promoted hematoma absorption. The protective effect of RXR-a activation was at least partly mediated by modulating M1/M2 macrophage polarization through PPAR-y signaling.

$\mathrm{ICH}$ is a cerebrovascular disease linked to high mortality and morbidity rates [28]. A hematoma within brain parenchyma leads to secondary injuries and severe neurological deficits [29]. To date, the efficient removal of the hematoma is a clinically promising intervention for intracerebral hemorrhage [30, 31]. Successful elimination of deposited blood and apoptotic cells by phagocytosis is essential for the resolution of inflammation [4]. RXR is a member of the superfamily of nuclear hormone receptors and consists of three distinct functional domains: an amino-terminal domain, involved in its ligandindependent basal transcriptional activity, a DNA-binding domain (DBD), and a ligand-binding domain (LBD). The LBD contains the ligand-binding regions that coordinate the formation of dimers and the recruiting of transcriptional coregulators [32]. RXR mediates their ligand-dependent transcriptional activities via LBD and DBD. It regulates metabolic and immune responses, including the regulation of macrophage immune phenotypes and the clearance of apoptotic cells $[13,33]$. Additionally, several studies have highlighted that RXR indeed played an important role in optimizing the phagocytic function in the CNS [34, 35]. Despite the role of RXR-a have been studied for years, little is known about the role of RXR-a in the pathological processes following ICH. 
Therefore, in the first part of this study, we assessed the effects of RXR-a in the pathological process of $\mathrm{ICH}$. RXR-a agonist bexarotene can significantly attenuate neurological function, which is negatively related to the volume of the existent hematoma after ICH in humans and rodents [36, 37]. Additionally, bexarotene significantly promoted hematoma resolution in the experimental mice model, which was consistent with the results of Chang et al. [38]. All of the above data suggest that the activation of RXR-a promoted hematoma clearance while conferring a neuroprotective effect against $\mathrm{ICH}$. However, the mechanisms underlying the protective effects of RXR-a remain unclear.

Microglia are vital to maintaining brain homeostasis, acting as phagocytes to scavenge for debris in the CNS. Targeting microglia may be an ideal therapeutic strategy for stroke [10, 39]. Besides, immunofluorescence staining showed that RXR-a was expressed in microglia/macrophages, astrocytes, and neurons. Notably, recent studies have demonstrated that PPAR-y in microglia/macrophages can increase phagocytosis and modulate inflammation by downregulating the expression of proinflammatory mediators expression $[31,40]$. Furthermore, to activate transcription, PPAR-y need to heterodimerize with RXR-a, which is an important signaling hub in nuclear receptor controlled transcription [41, 42]. More importantly, recent studies have confirmed that the PPAR-y signaling pathway is upregulated after exposure to bexarotene, suggesting an eminent role for PPAR- $\gamma$ in RXR-a transcriptional regulation [21, 35]. Mechanism underlying RXR transcriptional activities are related to its nucleoplasmic sublocalization to the nuclear splicing factor compartment [43]. Yasmin et al. reported that enhanced RXR nuclear translocation was enabled by importin- $\beta$ after RXR activation [44]. Given the essential role of RXR- $\alpha$ in regulating PPAR- $y$ transcription and the expression of RXR- $\alpha$ observed in microglia/macrophages after ICH, we hypothesize that RXR-a acts as an important regulator mediating the nuclear translocation of PPAR-y.

Therefore, we then investigated the nuclear translocation of RXR- $a$ and PPAR- $y$ after ICH. As a specific and irreversible inhibitor of PPAR- $\gamma$, we used GW9662 in the next experiment, which inactivated PPAR- $\gamma$ through covalent modification and does not change RXR-a activity [45]. We noted the enhanced translocation to the nucleus of RXR- $a$ and PPAR- $y$ after injury. Our findings were consistent with a previous study that reported enhanced translocation of RXR- $a$ and PPAR-y after traumatic brain injury [18]. Several studies on intracellular RXR shuttling were performed, showing that nuclear translocation of RXR was triggered by inflammatory cytokines such as IL-1 $\beta$, IL- 6 , and TNF-a [46, 47]. The local inflammatory response following injury may, therefore, contribute to the activation and subcellular translocation of the retinoid $X$ receptor, which had been shown to have anti-inflammatory properties [47]. Even though the translocation of RXR- $a$ and PPAR- $y$ into the nucleus was increased after ICH, this may not be sufficient to reduce the neuroinflammation after ICH. The pharmacological activation of RXR-a may further potentiate protective pathways against neuroinflammation after $\mathrm{ICH}$. We found that bexarotene significantly increased the nuclear translocation of RXR- $a$ and PPAR- $\gamma$, while the PPAR- $\gamma$ inhibitor GW9662 reversed this effect of PPAR-y. Notably, RXR- $a$ and PPAR- $y$ showed a similar trend towards nuclear translocation, and pharmacologically activating RXR-a could further promote their nuclear translocation, suggesting that RXR-a could synergistically with PPAR-y through its interaction with it. 
Various mechanisms are involved in the regulation of tissue healing, hematoma removal, and inflammation resolution. One of the essential mechanisms, especially concerning the neuroinflammation that is incurred by post-hemorrhagic secondary brain injuries, is related to the conversion of the classical activation phenotype (M1) to the alternative activation phenotype (M2) [10]. In the CNS, M2-like microglia/macrophages are efficient phagocytes [48]. Nuclear receptors play a key role in balancing M1/M2 polarization and controlling phagocytosis by regulating the transcription of functional genes [41]. It was demonstrated that PPAR- $y$ is an important transcriptional factor that plays a crucial role in switching on M1-like or M2-like marker genes, and thereby mediating the priming of microglia/macrophages towards M2 polarization [49]. Additionally, PPAR-y activation mediates the transcription of downstream genes, such as ABCA1 and Cd36, which are also considered as biomarkers of scavenger receptors that contribute to the phagocytic ability of microglia/macrophages $[3,35]$. A previous study has shown that the activation of the RXR/PPAR heterodimer exerts neuroprotective effects by modulating microglia polarization [18]. Given the involvement of RXR- $a$ and PPAR- $y$ in microglia/macrophages-type cell polarization and differentiation, we assessed the expression of M1/M2 markers in microglia/macrophages. M1 marker proteins (iNOS, TNF-a) and M2 marker protein (ARG1) were used, revealing that microglia/macrophages were significantly polarized into the classical activation phenotype (M1) after ICH. This finding was consistent with a previous study [18], as our data indicated that the activation of RXR-a could inhibit M1 activation and driving microglia/macrophages towards the M2 phenotype while PPAR-y inhibitor GW9662 reversed these effects. In the rest of this study, we further explored the role of PPAR-y in neurological function and hematoma volume in response to RXR-a activation after ICH. Consistent with our previous result, RXR-a agonists significantly alleviated neurological dysfunction and promoted hematoma resolution. However, all these beneficial effects were abolished by PPAR-y inhibitor GW9662. Taken together, we deduced that the activation of RXR-a promoted microglia/macrophages polarization towards the M2 phenotype through PPAR-y after ICH, therefore facilitating hematoma clearance and improving neurological function.

However, our research has some undeniable limitations. Firstly, as aforementioned, RXR-a expression was also observed in neurons and astrocytes, suggesting it may play a potential role in other cells. Secondly, in addition to forming heterodimers with PPAR- $y$, recent findings indicated that a separate RXR-a homodimer could also affect the immune system's function and participate in the regulation of innate immunity [50]. We solely focused on the RXR-a/PPAR-y signaling pathway in the current study. Lastly, resident microglia and peripheral microglia may both contribute to neuroinflammation; however, which of these mediate the beneficial effects has not yet been assessed [51]. Therefore, further efforts are required to obtain more details with regard to other possible mechanisms mediated by RXR-a in ICH.

In summary, our data indicated that pharmacological activation of RXR-a promoted hematoma clearance, improved neurological function, and polarized microglia/macrophages towards the M2 phenotype via regulation of the nuclear translocation of PPAR-y after ICH. Altogether, the current study supports the notion that targeting RXR-a might be a novel and promising therapeutic strategy for $\mathrm{ICH}$. 


\section{List Of Abbreviations}

BCA Bicinchoninic acid

CNS Central nervous system

DBD DNA-binding domain

DMSO Dimethyl sulfoxide

ELISA Enzyme-linked immunosorbent assay

$\mathrm{ICH} \quad$ Intracerebral hemorrhage

LBD Ligand-binding domain

MRI Magnetic resonance imaging

PPAR- $\gamma \quad$ Peroxisome proliferator-activated receptor- $\gamma$

RXR-a Retinoid X receptor-a

\section{Declarations}

Availability of Data and Materials: The datasets used and/or analyzed during the current study are available from the corresponding author on reasonable request.

Acknowledgments: Part of the figures were created using BioRender (www.BioRender.com), to which we are grateful. And we would like to thank Dr. Peiyu Huang for his expertise and assistance in radiology and imaging. This manuscript has been submitted to Research Square as a preprint in the link below: https://www.researchsquare.com/article/rs-67854/v1.

Authors' contributions: CRX, HJC, SJZ, CJS, and XLX performed the ICH model and Western blots. YCP, $J F Z$, and $\mathrm{HHZ}$ prepared the figures. QY and YC performed the immunostaining. YL, GYZ, XJF, and FY performed the MRI scanning. $\mathrm{HZ}$ and $\mathrm{HJC}$ performed data analysis. $\mathrm{LBH}$ participated in the neurological tests and in polishing the manuscript. GC and JRL designed experiments. CRX, HJC, and SJZ contributed to the writing and editing of the manuscript. All authors read and approved the manuscript.

Funding: This work was supported by the National Key R\&D Program of China (2018YFC1312600, 2018YFC1312603), the Key Research and Development Project of Zhejiang Province (No.2018C03011), National Natural Science Foundation of China (No.81771246, 81971099, 81870908), Scientific Research Fund of Zhejiang Provincial Education Department (No.Y201941838).

Ethics approval and consent to participate: All procedures involved animals were conformed to the Guide for the Care and Use of Laboratory Animals of the National Institutes of Health and were approved by the 
Institutional Animal Care and Use Committee of Zhejiang University.

Consent for publication: Not applicable.

Competing Interests: The authors declare that they have no competing interests.

\section{References}

[1] Qureshi Al, Mendelow AD, Hanley DF. Intracerebral haemorrhage. Lancet 2009, 373: 1632-1644.

[2] Kim JY, Bae HJ. Spontaneous Intracerebral Hemorrhage: Management. J Stroke 2017, 19: 28-39.

[3] Lan X, Han X, Li Q, Yang QW, Wang J. Modulators of microglial activation and polarization after intracerebral haemorrhage. Nat Rev Neurol 2017, 13: 420-433.

[4] Zhao X, Grotta J, Gonzales N, Aronowski J. Hematoma resolution as a therapeutic target: the role of microglia/macrophages. Stroke 2009, 40: S92-94.

[5] Mendelow AD, Gregson BA, Fernandes HM, Murray GD, Teasdale GM, Hope DT, et al. Early surgery versus initial conservative treatment in patients with spontaneous supratentorial intracerebral haematomas in the International Surgical Trial in Intracerebral Haemorrhage (STICH): a randomised trial. Lancet 2005, 365: 387-397.

[6] Mendelow AD, Gregson BA, Rowan EN, Murray GD, Gholkar A, Mitchell PM, et al. Early surgery versus initial conservative treatment in patients with spontaneous supratentorial lobar intracerebral haematomas (STICH II): a randomised trial. Lancet 2013, 382: 397-408.

[7] Wilkinson DA, Keep RF, Hua Y, Xi G. Hematoma clearance as a therapeutic target in intracerebral hemorrhage: From macro to micro. J Cereb Blood Flow Metab 2018, 38: 741-745.

[8] Xiong XY, Liu L, Yang QW. Functions and mechanisms of microglia/macrophages in neuroinflammation and neurogenesis after stroke. Prog Neurobiol 2016, 142: 23-44.

[9] Qin C, Zhou LQ, Ma XT, Hu ZW, Yang S, Chen M, et al. Dual Functions of Microglia in Ischemic Stroke. Neurosci Bull 2019, 35: 921-933.

[10] Tschoe C, Bushnell CD, Duncan PW, Alexander-Miller MA, Wolfe SQ. Neuroinflammation after Intracerebral Hemorrhage and Potential Therapeutic Targets. J Stroke 2020, 22: 29-46.

[11] Cherry JD, Olschowka JA, O'Banion MK. Neuroinflammation and M2 microglia: the good, the bad, and the inflamed. Journal of Neuroinflammation 2014, 11: 98.

[12] Prufer K, Barsony J. Retinoid X receptor dominates the nuclear import and export of the unliganded vitamin D receptor. Mol Endocrinol 2002, 16: 1738-1751. 
[13] Roszer T, Menendez-Gutierrez MP, Cedenilla M, Ricote M. Retinoid X receptors in macrophage biology. Trends Endocrinol Metab 2013, 24: 460-468.

[14] Safi R, Muramoto GG, Salter AB, Meadows S, Himburg H, Russell L, et al. Pharmacological Manipulation of the RAR/RXR Signaling Pathway Maintains the Repopulating Capacity of Hematopoietic Stem Cells in Culture. Molecular Endocrinology 2009, 23: 188-201.

[15] Nagy L, Szanto A, Szatmari I, Széles L. Nuclear Hormone Receptors Enable Macrophages and Dendritic Cells to Sense Their Lipid Environment and Shape Their Immune Response. Physiological Reviews 2012, 92: 739-789.

[16] Cramer PE, Cirrito JR, Wesson DW, Lee CY, Karlo JC, Zinn AE, et al. ApoE-directed therapeutics rapidly clear beta-amyloid and reverse deficits in AD mouse models. Science 2012, 335: 1503-1506.

[17] Dheer Y, Chitranshi N, Gupta V, Abbasi M, Mirzaei M, You Y, et al. Bexarotene Modulates Retinoid-XReceptor Expression and Is Protective Against Neurotoxic Endoplasmic Reticulum Stress Response and Apoptotic Pathway Activation. Mol Neurobiol 2018, 55: 9043-9056.

[18] He J, Liu H, Zhong J, Guo Z, Wu J, Zhang H, et al. Bexarotene protects against neurotoxicity partially through a PPARY-dependent mechanism in mice following traumatic brain injury. Neurobiology of Disease 2018, 117: 114-124.

[19] Sansing LH, Kasner SE, McCullough L, Agarwal P, Welsh FA, Kariko K. Autologous blood injection to model spontaneous intracerebral hemorrhage in mice. J Vis Exp 2011.

[20] Gniadecki R, Assaf C, Bagot M, Dummer R, Duvic M, Knobler R, et al. The optimal use of bexarotene in cutaneous T-cell lymphoma. Br J Dermatol 2007, 157: 433-440.

[21] Zhong J, Cheng C, Liu H, Huang Z, Wu Y, Teng Z, et al. Bexarotene protects against traumatic brain injury in mice partially through apolipoprotein E. Neuroscience 2017, 343: 434-448.

[22] Flores JJ, Klebe D, Rolland WB, Lekic T, Krafft PR, Zhang JH. PPARgamma-induced upregulation of CD36 enhances hematoma resolution and attenuates long-term neurological deficits after germinal matrix hemorrhage in neonatal rats. Neurobiol Dis 2016, 87: 124-133.

[23] He J, Liu H, Zhong J, Guo Z, Wu J, Zhang H, et al. Bexarotene protects against neurotoxicity partially through a PPARgamma-dependent mechanism in mice following traumatic brain injury. Neurobiol Dis 2018, 117: 114-124.

[24] Jing C, Bian L, Wang M, Keep RF, Xi G, Hua Y. Enhancement of Hematoma Clearance With CD47 Blocking Antibody in Experimental Intracerebral Hemorrhage. Stroke 2019, 50: 1539-1547.

[25] Chang CF, Goods BA, Askenase MH, Hammond MD, Renfroe SC, Steinschneider AF, et al. Erythrocyte efferocytosis modulates macrophages towards recovery after intracerebral hemorrhage. J Clin Invest 
2018, 128: 607-624.

[26] Anguissola S, McCormack WJ, Morrin MA, Higgins WJ, Fox DM, Worrall DM. Pigment epitheliumderived factor (PEDF) interacts with transportin SR2, and active nuclear import is facilitated by a novel nuclear localization motif. PLoS One 2011, 6: e26234.

[27] Peng Y, Zhuang J, Ying G, Zeng H, Zhou H, Cao Y, et al. Stimulator of IFN genes mediates neuroinflammatory injury by suppressing AMPK signal in experimental subarachnoid hemorrhage. Journal of Neuroinflammation 2020, 17: 165.

[28] Fogelholm R, Murros K, Rissanen A, Avikainen S. Long term survival after primary intracerebral haemorrhage: a retrospective population based study. J Neurol Neurosurg Psychiatry 2005, 76: 15341538.

[29] Keep RF, Hua Y, Xi G. Intracerebral haemorrhage: mechanisms of injury and therapeutic targets. Lancet Neurol 2012, 11: 720-731.

[30] Zhao XR, Gonzales N, Aronowski J. Pleiotropic role of PPARgamma in intracerebral hemorrhage: an intricate system involving Nrf2, RXR, and NF-kappaB. CNS Neurosci Ther 2015, 21: 357-366.

[31] Zhao X, Sun G, Zhang J, Strong R, Song W, Gonzales N, et al. Hematoma resolution as a target for intracerebral hemorrhage treatment: role for peroxisome proliferator-activated receptor gamma in microglia/macrophages. Ann Neurol 2007, 61: 352-362.

[32] Jin L, Li Y. Structural and functional insights into nuclear receptor signaling. Adv Drug Deliv Rev 2010, 62: 1218-1226.

[33] Núñez V, Alameda D, Rico D, Mota R, Gonzalo P, Cedenilla M, et al. Retinoid X receptor a controls innate inflammatory responses through the up-regulation of chemokine expression. Proceedings of the National Academy of Sciences 2010, 107: 10626-10631.

[34] Natrajan MS, de la Fuente AG, Crawford AH, Linehan E, Nuñez V, Johnson KR, et al. Retinoid X receptor activation reverses age-related deficiencies in myelin debris phagocytosis and remyelination. Brain 2015, 138: 3581-3597.

[35] Ting S-M, Zhao X, Sun G, Obertas L, Ricote M, Aronowski J. Brain Cleanup as a Potential Target for Poststroke Recovery. Stroke 2020, 51: 958-966.

[36] Broderick JP, Brott TG, Duldner JE, Tomsick T, Huster G. Volume of intracerebral hemorrhage. A powerful and easy-to-use predictor of 30-day mortality. Stroke 1993, 24: 987-993.

[37] MacLellan CL, Auriat AM, McGie SC, Yan RH, Huynh HD, De Butte MF, et al. Gauging recovery after hemorrhagic stroke in rats: implications for cytoprotection studies. J Cereb Blood Flow Metab 2006, 26: 1031-1042. 
[38] Chang CF, Massey J, Osherov A, Angenendt da Costa LH, Sansing LH. Bexarotene Enhances Macrophage Erythrophagocytosis and Hematoma Clearance in Experimental Intracerebral Hemorrhage. Stroke 2020, 51: 612-618.

[39] Umpierre AD, Wu LJ. Microglia Research in the 100th Year Since Its Discovery. Neurosci Bull 2020, 36: 303-306.

[40] Wang Y, Chen Q, Tan Q, Feng Z, He Z, Tang J, et al. Simvastatin accelerates hematoma resolution after intracerebral hemorrhage in a PPARgamma-dependent manner. Neuropharmacology 2018, 128: 244254.

[41] Roszer T. Transcriptional control of apoptotic cell clearance by macrophage nuclear receptors. Apoptosis 2017, 22: 284-294.

[42] Dubuquoy L, Dharancy S, Nutten S, Pettersson S, Auwerx J, Desreumaux P. Role of peroxisome proliferator-activated receptor gamma and retinoid $\mathrm{X}$ receptor heterodimer in hepatogastroenterological diseases. Lancet 2002, 360: 1410-1418.

[43] Lefebvre P, Benomar Y, Staels B. Retinoid X receptors: common heterodimerization partners with distinct functions. Trends Endocrinol Metab 2010, 21: 676-683.

[44] Yasmin R, Williams RM, Xu M, Noy N. Nuclear import of the retinoid X receptor, the vitamin D receptor, and their mutual heterodimer. J Biol Chem 2005, 280: 40152-40160.

[45] Leesnitzer LM, Parks DJ, Bledsoe RK, Cobb JE, Collins JL, Consler TG, et al. Functional consequences of cysteine modification in the ligand binding sites of peroxisome proliferator activated receptors by GW9662. Biochemistry 2002, 41: 6640-6650.

[46] Mey J, Schrage K, Wessels I, Vollpracht-Crijns I. Effects of inflammatory cytokines IL-1 beta, IL-6, and TNFalpha on the intracellular localization of retinoid receptors in Schwann cells. Glia 2007, 55: 152-164.

[47] van Neerven S, Mey J. RAR/RXR and PPAR/RXR Signaling in Spinal Cord Injury. PPAR Research 2007, 2007: 029275.

[48] Cherry JD, Olschowka JA, O'Banion MK. Neuroinflammation and M2 microglia: the good, the bad, and the inflamed. J Neuroinflammation 2014, 11: 98.

[49] Hu X, Leak RK, Shi Y, Suenaga J, Gao Y, Zheng P, et al. Microglial and macrophage polarization-new prospects for brain repair. Nat Rev Neurol 2015, 11: 56-64.

[50] Nunez V, Alameda D, Rico D, Mota R, Gonzalo P, Cedenilla M, et al. Retinoid X receptor alpha controls innate inflammatory responses through the up-regulation of chemokine expression. Proc Natl Acad Sci U S A 2010, 107: 10626-10631. 


\section{Figures}

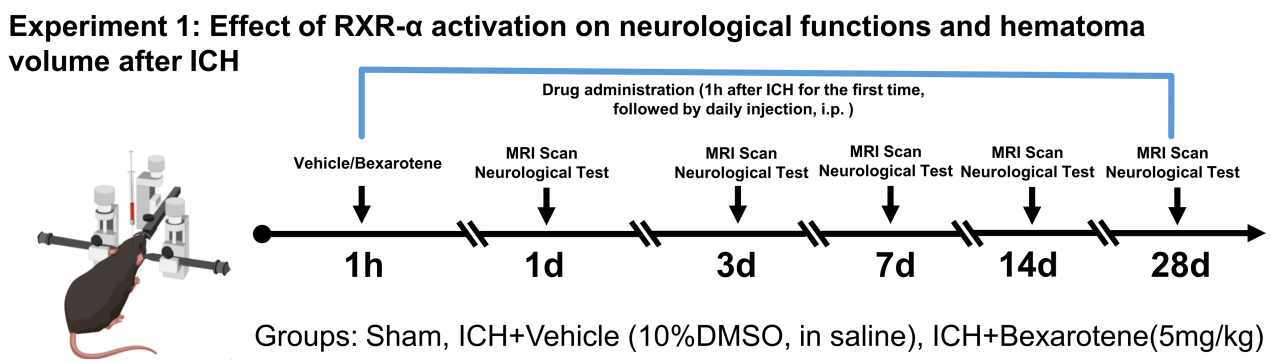

Experiment 2: Expression of RXR- $\alpha$ and PPAR-y after ICH
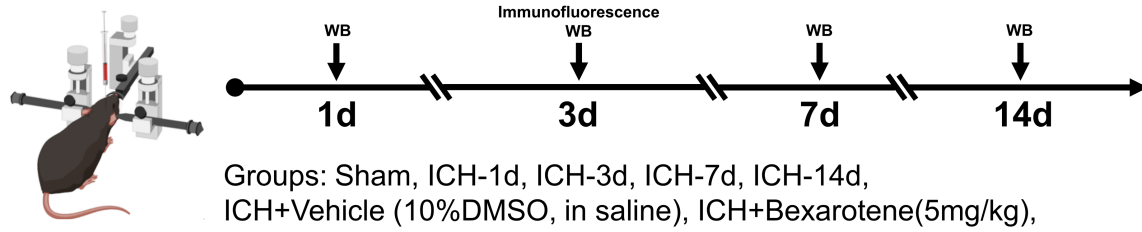

Groups: Sham, ICH-1d, ICH-3d, ICH-7d, ICH-14d,

$\mathrm{ICH}+$ Vehicle (10\%DMSO, in saline), $\mathrm{ICH}+$ Bexarotene $(5 \mathrm{mg} / \mathrm{kg})$,

$\mathrm{ICH}+$ Bexarotene $(5 \mathrm{mg} / \mathrm{kg})+$ Vehicle $(10 \% \mathrm{DMSO}$, in saline $)$,

$\mathrm{ICH}+$ Bexarotene $(5 \mathrm{mg} / \mathrm{kg})+\mathrm{GW} 9662(4 \mathrm{mg} / \mathrm{kg})$

Experiment 3: Mechanisms of the protective effect of RXR- $\alpha$ activation after ICH

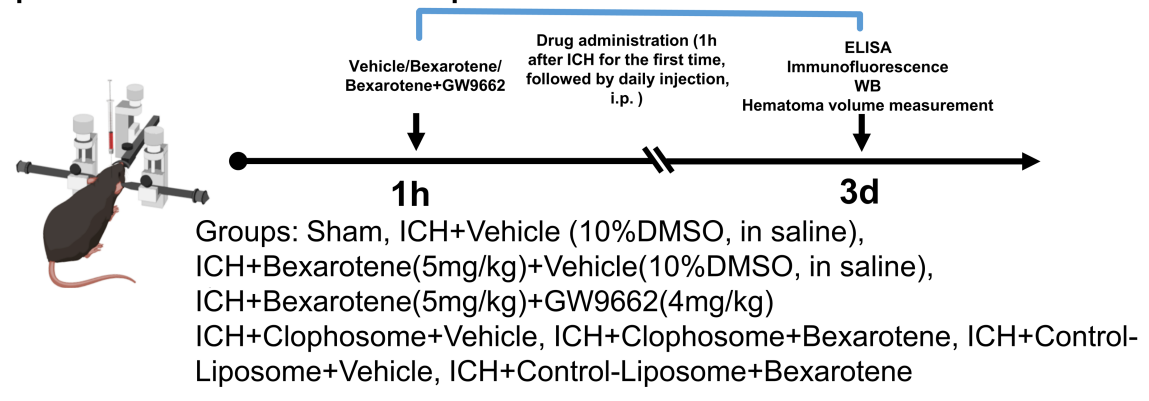

Experiment 4: Roles of PPAR-y in neurological functions and hematoma volume after

$\mathrm{ICH}$
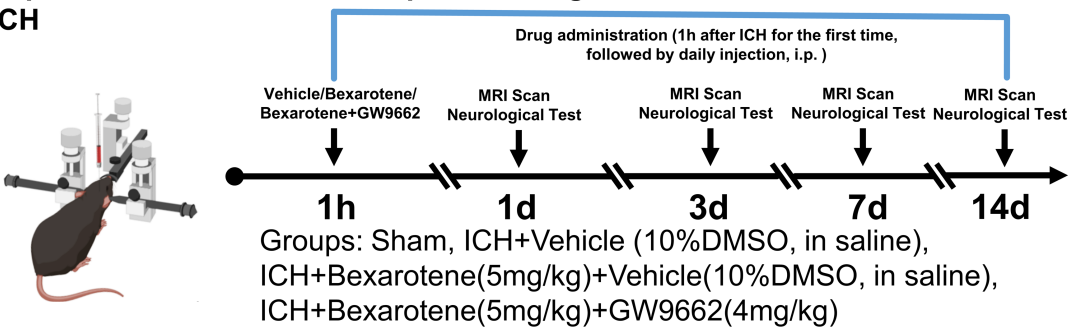

Figure 1 
Experimental design and animal groups. Part of the figure was created using BioRender (www.biorender.com).

A

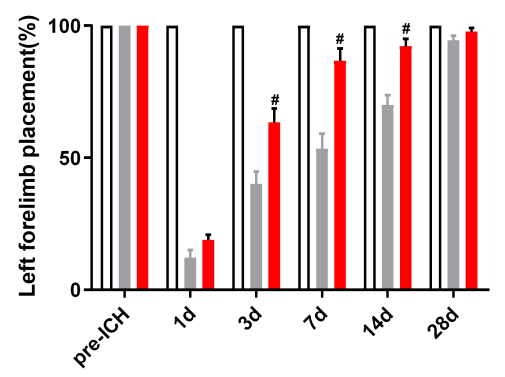

C

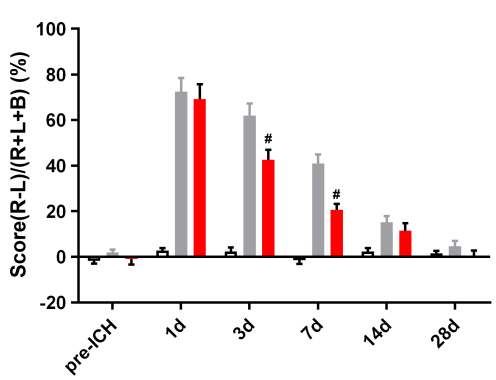

B

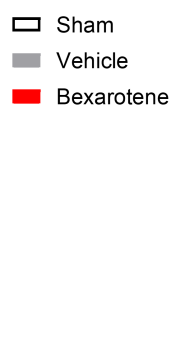

$\square$ Sham

- Vehicle

- Bexarotene
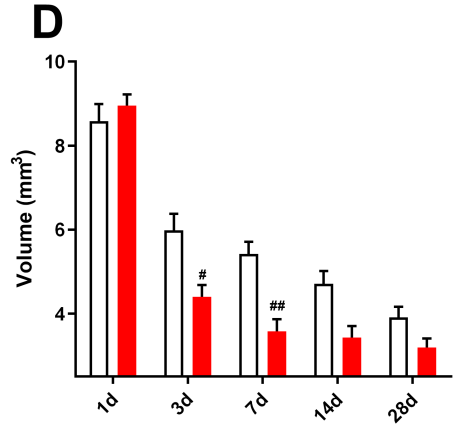

$\square$ Sham

Vehicle

- Bexarotene

E

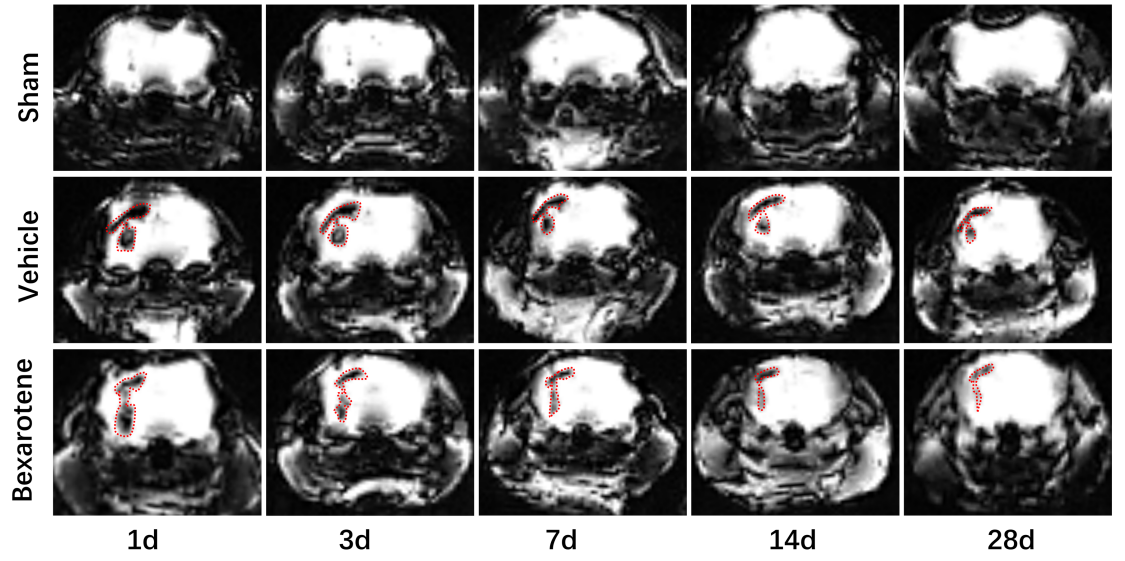

Figure 2

Pharmacological activation of RXR-a significantly alleviated neurological dysfunction and promoted hematoma clearance after ICH. Quantification of neurological function with, (A) forelimb placement test, (B) corner turn test, and (C) cylinder test at 1, 3, 7, 14, and 28 days. $n=9 /$ group. Data are represented as 
mean \pm SEM. \#p $<0.05$ versus ICH + vehicle group. D: Quantitative analysis of hematoma volumes. $\mathrm{n}=$ 6 /group. Data are represented as mean \pm SEM. \#p $<0.05$ versus $\mathrm{ICH}+$ vehicle group. \#\#p $<0.001$ versus $\mathrm{ICH}+$ vehicle group. $\mathrm{E}: \mathrm{T} 2{ }^{*}$-weighted MRI scans (coronal sections) showing the sham group image and the changes in hematoma volumes overtime after $\mathrm{ICH}$. The red dotted lines denote the hematoma.
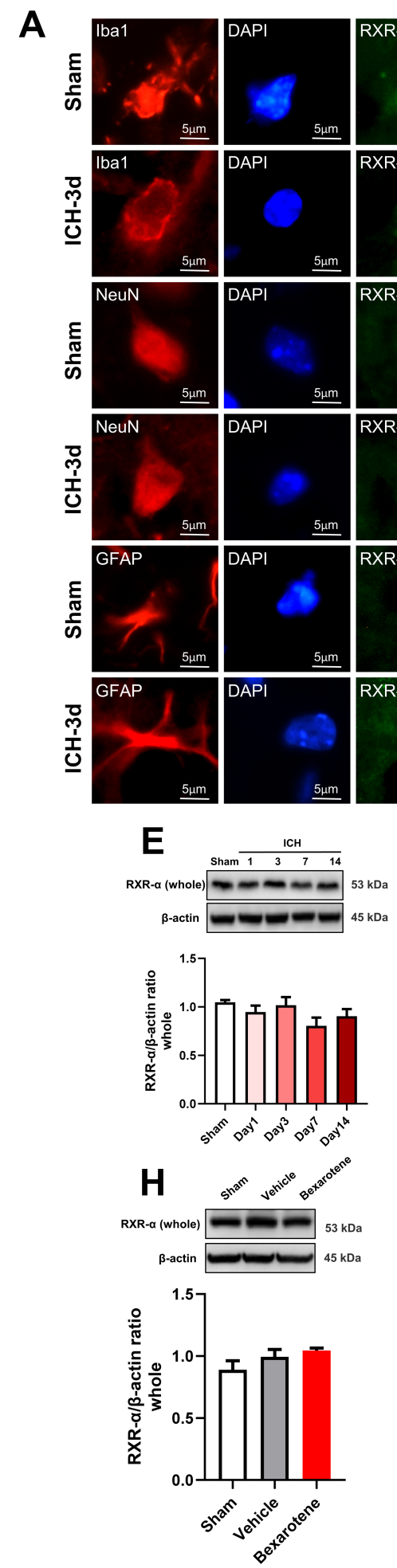
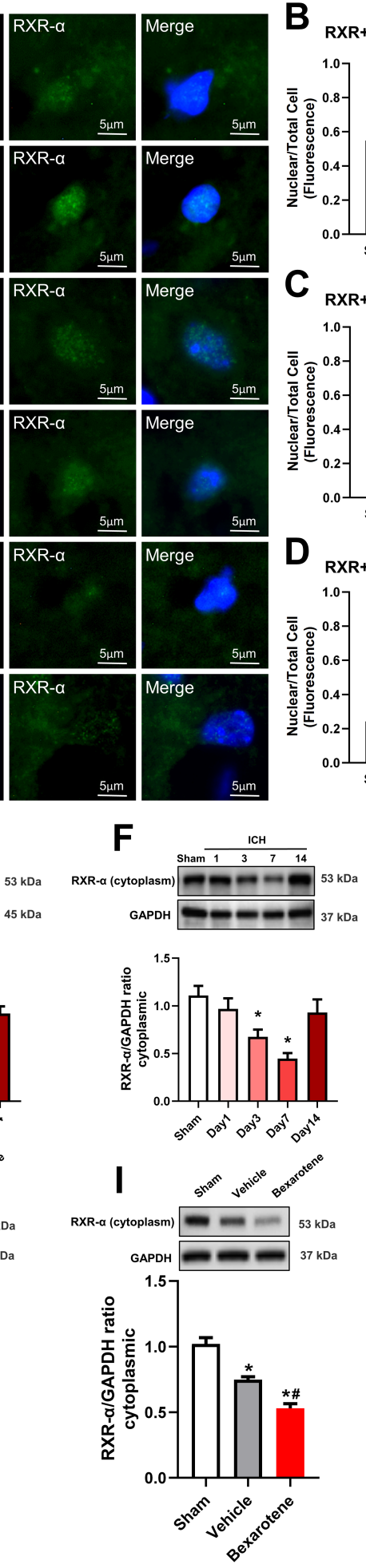

$\mathrm{B}_{\mathrm{RXR}+\mathrm{Hb} 1+\mathrm{cells}}$

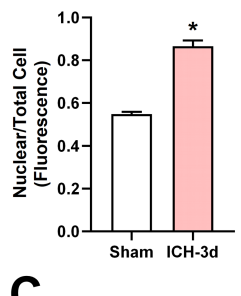

C RXR+NeuN+ cells

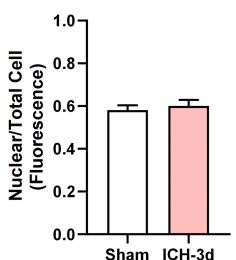

$D_{\text {RXR+GFAP+ cells }}$

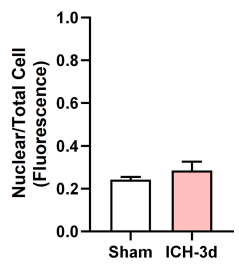

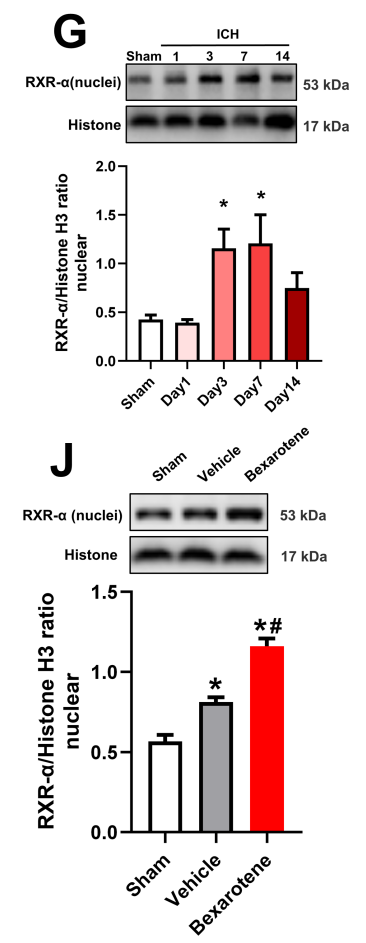

Figure 3 
Bexarotene promoted nuclear translocation of RXR-a in microglia/macrophages. A: Representative microphotographs of immunofluorescence double staining showing the RXR-a (green) with NeuN, GFAP, and Iba-1 (red) in the sham group and ICH $72 \mathrm{~h}$ group. Scale bar $=5 \mu \mathrm{m} . \mathrm{n}=6 /$ group. B: Calculation of the ratio of the nucleus and the whole-cell fluorescence of microglia/macrophages expressing RXR- $a . n=$ $6 /$ group. Data are represented as mean \pm SEM. ${ }^{*} p<0.05$ versus sham. C: Calculation of the ratio of the nucleus and the whole-cell fluorescence of neurons expressing RXR-a. $n=6 /$ group. Data are represented as mean \pm SEM. D: Calculation of the ratio of the nucleus and the whole-cell fluorescence of astrocytes expressing RXR-a. $n=6 /$ group. Data are represented as mean \pm SEM. E: Representative Western blotting images and quantitative analyses of RXR-a in the whole cells of ipsilateral basal ganglia after ICH by Western blot. $n=6$ /group. F: Representative Western blotting images and quantitative analyses of RXR-a in the cytoplasm of ipsilateral basal ganglia after ICH by Western blot. $n=6 /$ group, mean $\pm S E M,{ }^{*} p<0.05$ versus sham. G: Representative Western blotting images and quantitative analyses of RXR-a in the nuclei of ipsilateral basal ganglia after ICH by Western blot. $n=6$ /group, mean $\pm S E M,{ }^{*} p<0.05$ versus sham. $H$ : Representative Western blotting images and quantitative analyses of RXR-a in the whole cells of ipsilateral basal ganglia after ICH. $n=6 /$ group. I: Representative Western blotting images and quantitative analyses of RXR-a in the cytoplasm of ipsilateral basal ganglia after ICH. $n=6 /$ group. Data are represented as mean \pm SEM. ${ }^{*} p<0.05$ versus sham, $\# p<0.05$ versus $\mathrm{ICH}+$ vehicle group. J: Representative Western blotting images and quantitative analyses of RXR- $a$ in the nuclei of ipsilateral basal ganglia after ICH. $n=6 /$ group. Data are represented as mean \pm SEM. ${ }^{\star} p<0.05$ versus sham, $\# p<$ 0.05 versus $\mathrm{ICH}+$ vehicle group. 
A
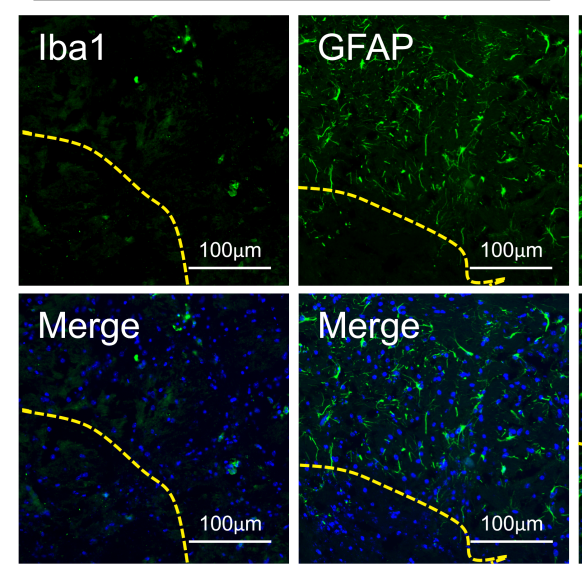

B

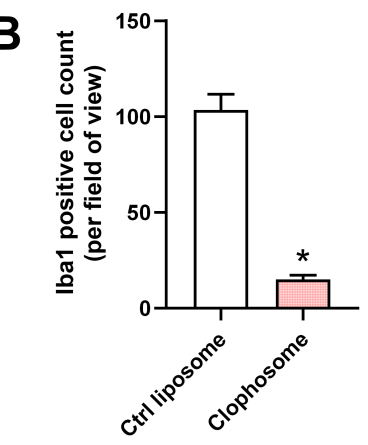

Ctrl Liposome
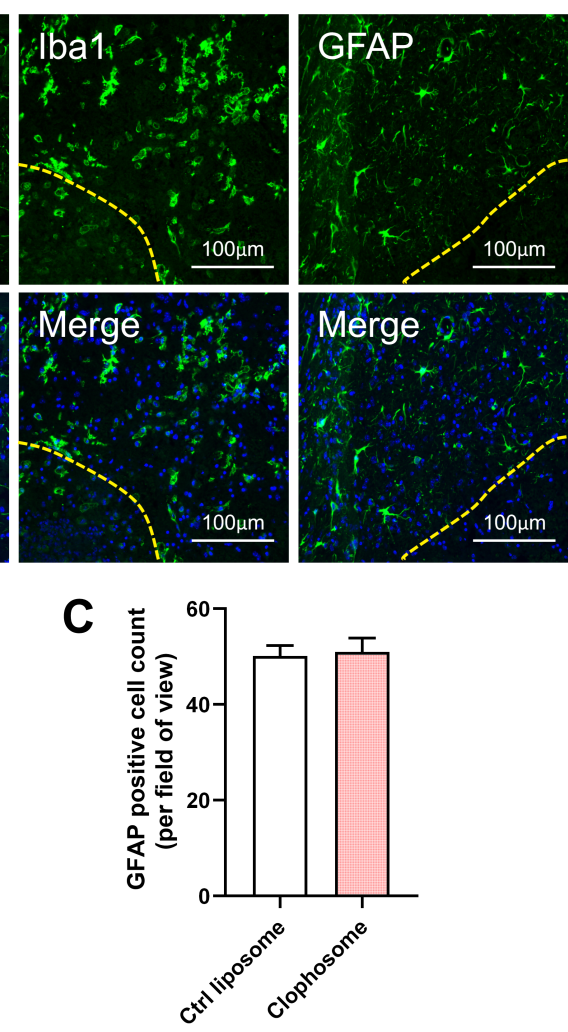

D

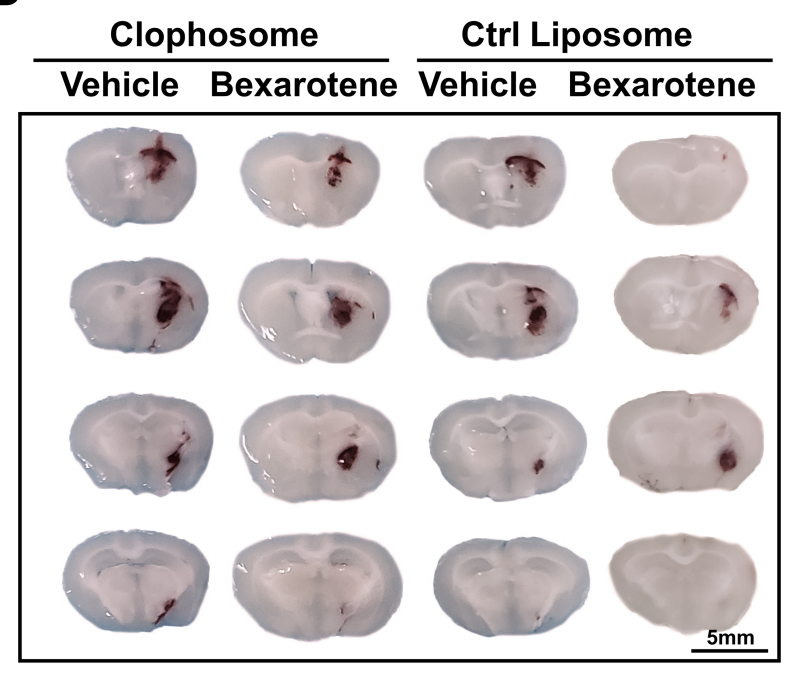

$\mathbf{E}$

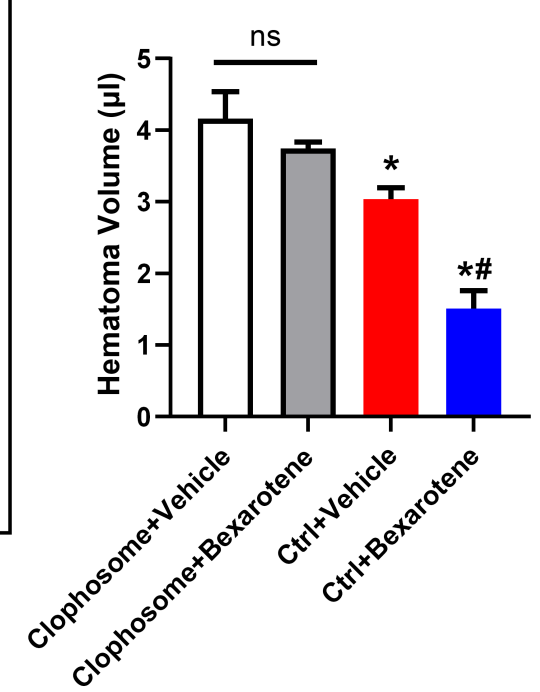

\section{Figure 4}

Depletion of microglia/macrophages inhibited bexarotene-mediated hematoma clearance. A:

Representative microphotographs of Iba1 positive and GFAP positive cells around the hematoma. Scale bar $=100 \mu \mathrm{m}$. Dotted lines mark the hematoma boundary in the fluorescent images. B: Quantitative analysis of Iba1 positive cells, Data are represented as mean \pm SEM. ${ }^{*} p<0.05$ versus control group. C: Quantitative analysis of GFAP positive cells. Data are represented as mean \pm SEM. D: Representative 
brain coronal sections show hematoma after treated with Clophosome + vehicle, Clophosome + bexarotene, control + vehicle, control + bexarotene at days 3. Scale bar $=5 \mathrm{~mm}$. E: Quantification of hematoma volume. $n=6$ / group. Data are represented as mean \pm SEM. ${ }^{*} p<0.05$ versus Clophosome + vehicle, $\# p<0.05$ versus control + vehicle group.

A
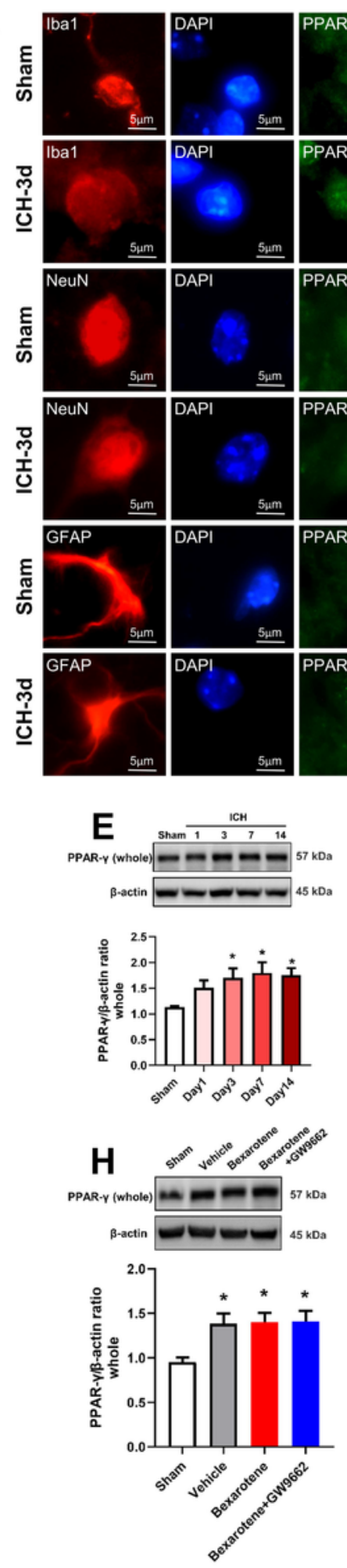

$B_{\text {PPAR+lba1+ cells }}$

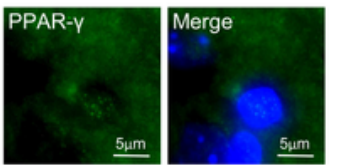

PPAR-Y
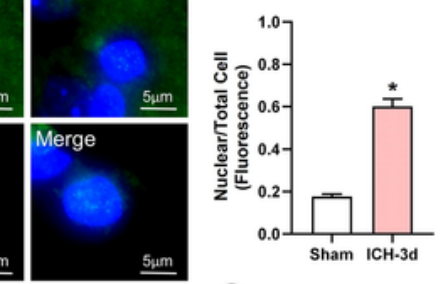

C ${ }_{\text {PPAR }+ \text { Neun }+ \text { cells }}$
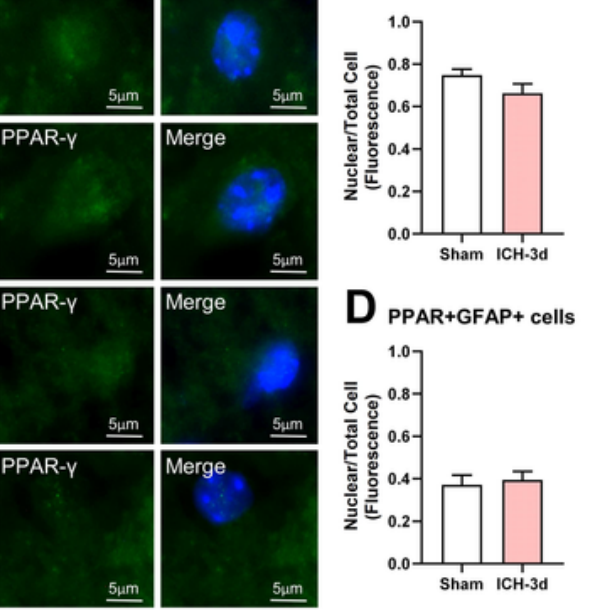
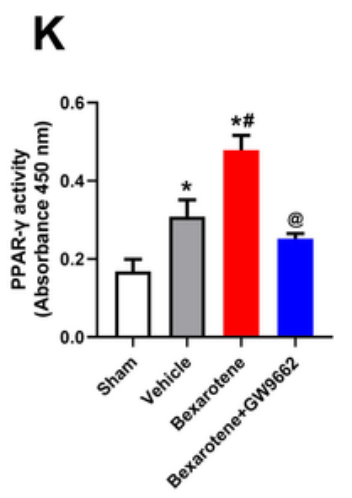
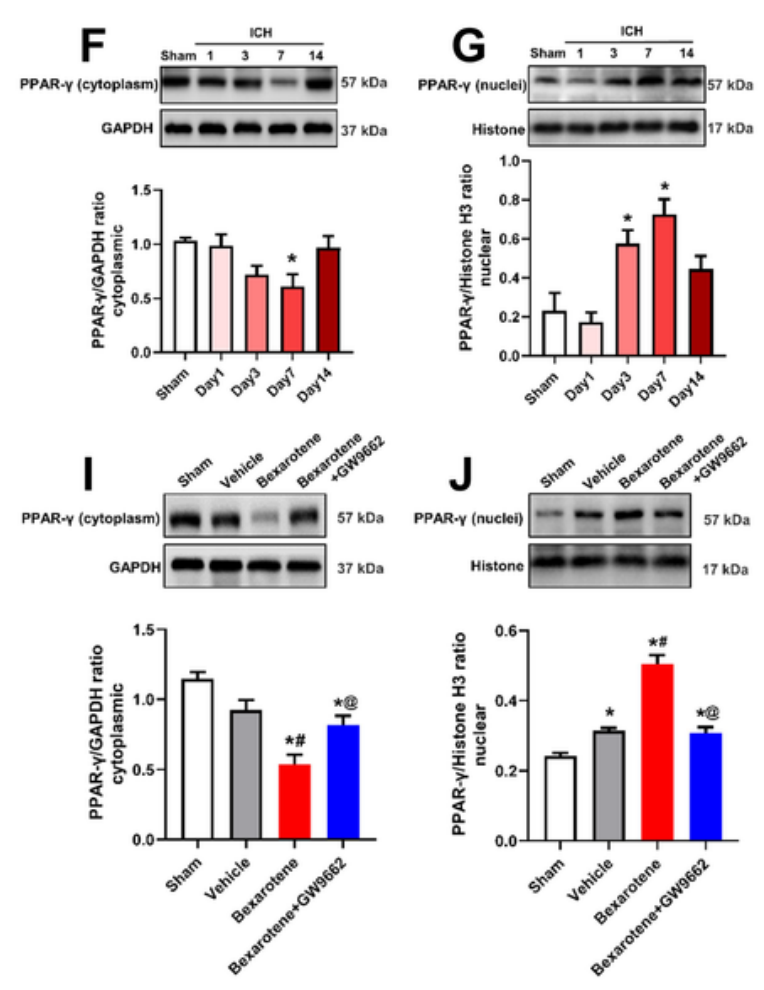

Figure 5 
Activation of RXR-a with bexarotene enhanced the nuclear translocation and transcription activities of PPAR-y. A: Representative microphotographs of immunofluorescence double staining showing the PPAR$\mathrm{Y}$ (green) with NeuN, GFAP, and Iba-1 (red) in the sham group and ICH $72 \mathrm{~h}$ group. Scale bar $=5 \mu \mathrm{m}$. $n=6 /$ group. B: Calculation of the ratio of the nucleus and the whole-cell fluorescence of microglia/macrophages expressing PPAR- $\gamma . n=6$ /group. Data are represented as mean \pm SEM. * $p<0.05$ versus sham. C: Calculation of the ratio of the nucleus and the whole-cell fluorescence of neurons expressing PPAR-y. $n=6 /$ group. Data are represented as mean \pm SEM. D: Calculation of the ratio of the nucleus and the whole-cell fluorescence of astrocytes expressing PPAR-y. $n=6 /$ group. Data are represented as mean \pm SEM. E: Representative Western blotting images and quantitative analyses of PPAR- $\gamma$ in the whole cells of ipsilateral basal ganglia after ICH by Western blot. $n=6 /$ group, mean \pm SEM, ${ }^{*} p<0.05$ versus sham. F: Representative Western blotting images and quantitative analyses of PPAR- $y$ in the cytoplasm of ipsilateral basal ganglia after ICH by Western blot. $n=6 /$ group, mean \pm SEM, ${ }^{*}<0.05$ versus sham. G: Representative Western blotting images and quantitative analyses of PPAR- $\gamma$ in nuclei of ipsilateral basal ganglia after ICH by Western blot. $n=6 /$ group, mean $\pm \mathrm{SEM},{ }^{*} \mathrm{p}<0.05$ versus sham. $\mathrm{H}$ : Representative Western blotting images and quantitative analyses of PPAR-y in the whole cells of ipsilateral basal ganglia after ICH. $n=6$ /group, mean \pm SEM, ${ }^{\star} p<0.05$ versus sham. I: Representative Western blotting images and quantitative analyses of PPAR-y in the cytoplasm of ipsilateral basal ganglia after $\mathrm{ICH} . \mathrm{n}=6$ /group. Data are represented as mean $\pm \mathrm{SEM}$. ${ }^{\star} \mathrm{p}<0.05$ versus sham, $\# p<0.05$ versus $\mathrm{ICH}+$ vehicle group, @p $<0.05$ versus $\mathrm{ICH}+$ bexarotene group. J: Representative Western blotting images and quantitative analyses of PPAR-y in nuclei of ipsilateral basal ganglia after ICH. $n=6 /$ group. Data are represented as mean \pm SEM. * $p<0.05$ versus sham, $\# p<0.05$ versus $\mathrm{ICH}+$ vehicle group, @p < 0.05 versus ICH + bexarotene group. K: Quantitative analyses of PPAR-y transcription activity at 3 days after ICH. $n=6$ /group. Data are represented as mean \pm SEM. ${ }^{\star} p<0.05$ versus sham, $\# p<0.05$ versus $\mathrm{ICH}$ + vehicle group. @P $<0.05$ versus $\mathrm{ICH}+$ bexarotene group. 
A
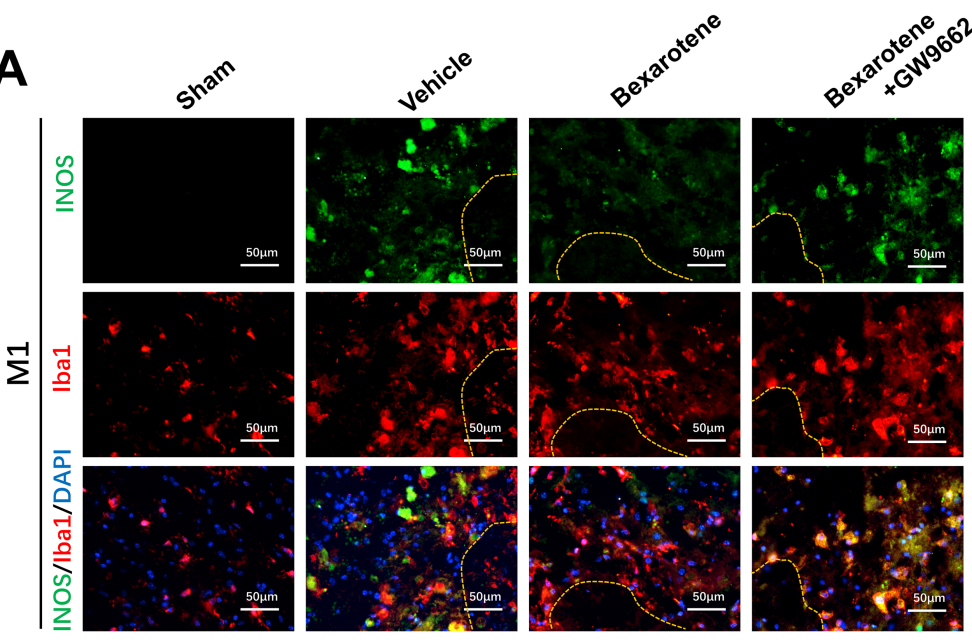

B
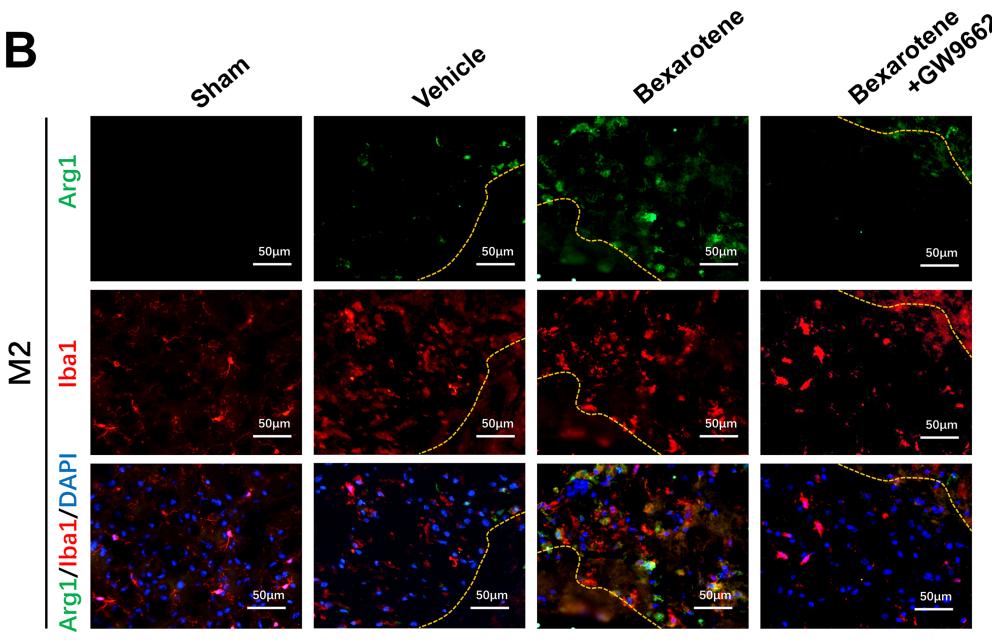

C

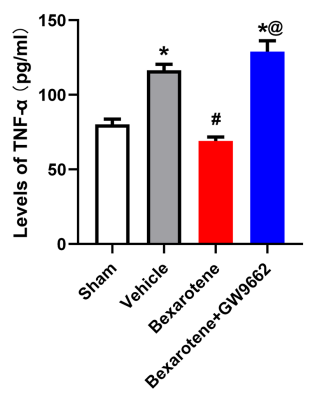

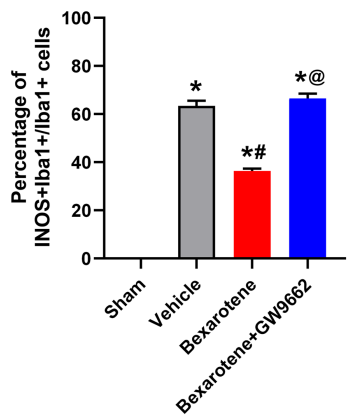

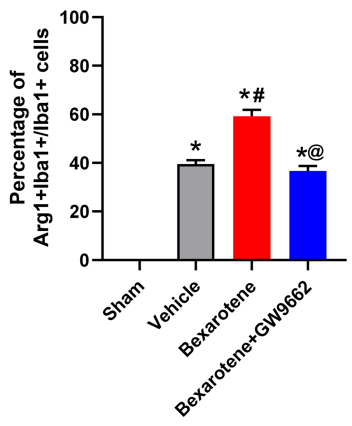

D

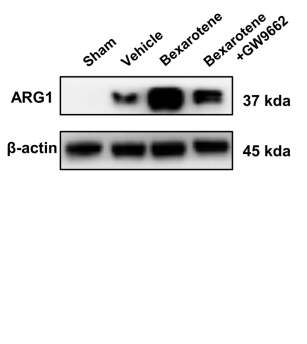

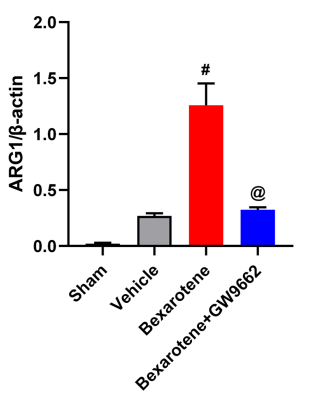

Figure 6

RXR-a activation regulates microglia/macrophages polarization through PPAR-ү. A: Representative microphotographs and quantitative analysis of iNOS positive (green) and Iba1positive (red). Scale bar = $50 \mu \mathrm{m} . \mathrm{n}=4$ /group. Dotted lines mark the hematoma boundary in the fluorescent images. Data are represented as mean \pm SEM. * $p<0.05$ versus sham, $\# p<0.05$ versus ICH + vehicle group, @p $<0.05$ versus $\mathrm{ICH}+$ bexarotene group. B: Representative microphotographs and quantitative analysis of ARG1 
positive (green) and Iba1-positive (red) cells. Scale bar $=50 \mu \mathrm{m} . n=4 /$ group. Dotted lines mark the hematoma boundary in the fluorescent images. Data are represented as mean $\pm S E M$. ${ }^{*} p<0.05$ versus sham, $\# \mathrm{p}<0.05$ versus ICH + vehicle group, @p $<0.05$ versus ICH + bexarotene group. C: Quantitative analyses of TNF-a at 3 days after ICH. $n=6$ /group. Data are represented as mean \pm SEM. ${ }^{*} p<0.05$ versus sham, $\# \mathrm{p}<0.05$ versus $\mathrm{ICH}+$ vehicle group. @P $<0.05$ versus $\mathrm{ICH}+$ bexarotene group. $\mathrm{D}$ :

Representative Western blotting images and quantitative analyses of ARG1 at 3 days after ICH. $n=$ 6 /group. Data are represented as mean \pm SEM. \#p $<0.05$ versus ICH + vehicle group. @p $<0.05$ versus $\mathrm{ICH}+$ bexarotene group. 
A

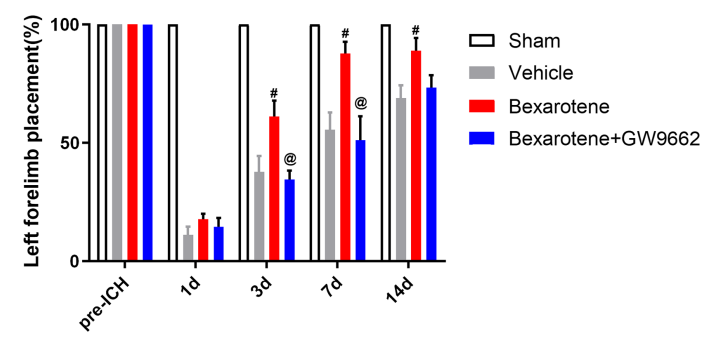

C

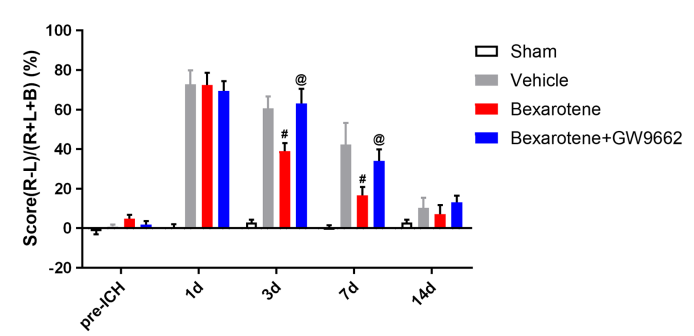

B

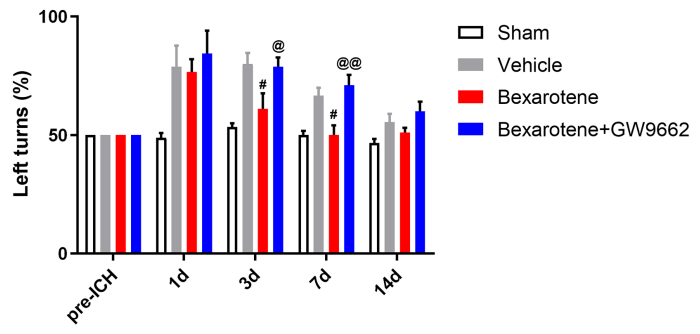

D

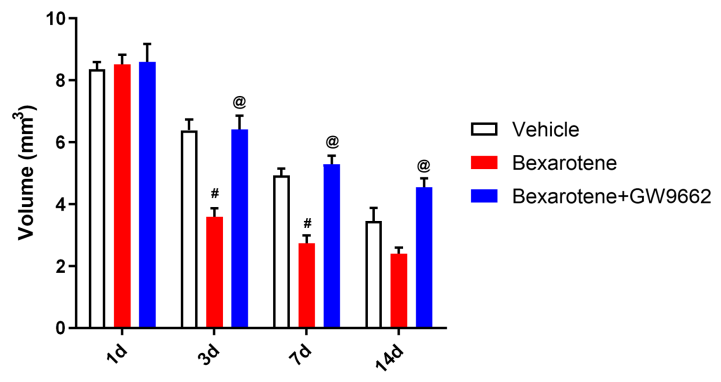

E
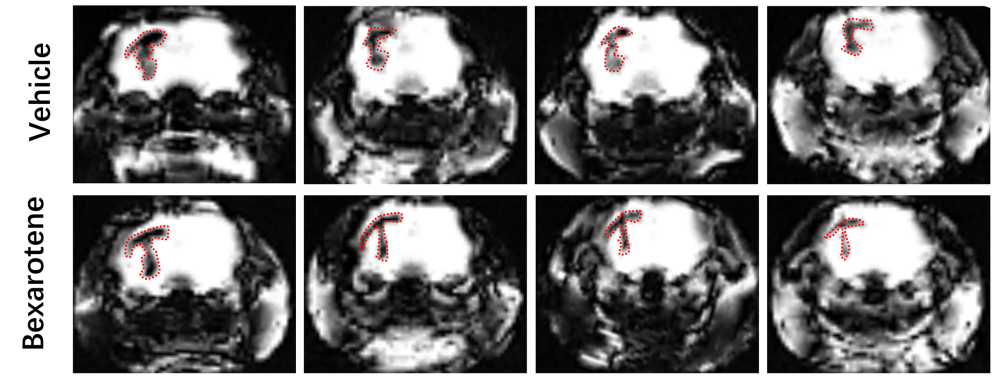

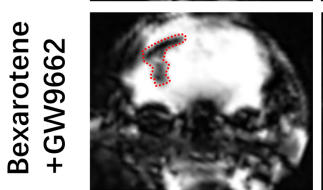

$1 d$

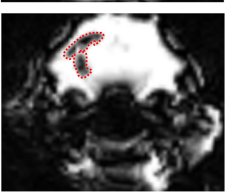

$3 d$

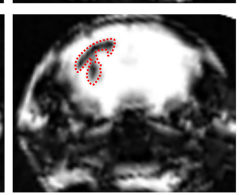

$7 d$
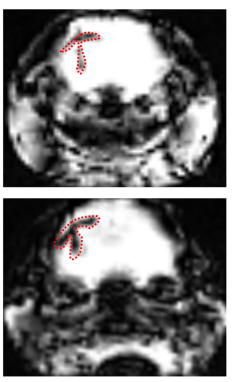

$14 d$

Figure 7

Role of PPAR-y on RXR-a-mediated neuroprotection and hematoma absorption against ICH.

Quantification of neurological function with (A) forelimb placement test, (B) corner turn test, and (C) cylinder test at $1,3,7$, and 14 days. $n=9$ /group. Data are represented as mean $\pm S E M$. $\# p<0.05$ versus ICH + vehicle group, @p $<0.05$ versus ICH + bexarotene group, @@p 0.01 versus ICH + bexarotene group. D: quantitative analysis of hematoma volumes. $n=6 /$ group. Data are represented as mean \pm 
SEM. \#p $<0.05$ versus ICH + vehicle group, @p $<0.05$ versus ICH + bexarotene group. E: Representative T2*-weighted MRI scans (coronal sections) showing the changes in hematoma volumes overtime after $\mathrm{ICH}$. The red dotted lines denote the hematoma. 